\title{
Low sensitivity of histology to predict the presence of microorganisms in suspected aseptic loosening of a joint prosthesis
}

\author{
Guillem Bori ${ }^{1}$, Alex Soriano ${ }^{2}$, Sebastián García ${ }^{1}$, Xavier Gallart ${ }^{1}$, Luis Casanova ${ }^{1}$, \\ Carme Mallofre ${ }^{3}$, Manel Almela ${ }^{4}$, José A Martínez ${ }^{2}$, Josep Riba ${ }^{1}$ and Josep Mensa ${ }^{2}$ \\ ${ }^{1}$ Department of Orthopaedic and Traumatology, Hospital Clínic, University of Barcelona, Barcelona, \\ Catalonia, Spain; ${ }^{2}$ Department of Infectious Diseases, Hospital Clinic, University of Barcelona, Barcelona, \\ Catalonia, Spain; ${ }^{3}$ Department of Pathology, Hospital Clínic, University of Barcelona, Barcelona, Catalonia, \\ Spain and ${ }^{4}$ Department of Microbiology, Hospital Clínic, University of Barcelona, Barcelona, Catalonia, \\ Spain
}

\begin{abstract}
Intraoperative histology has a high specificity and sensitivity when a septic prosthesis loosening is suspected. However, its usefulness to predict the presence of microorganisms when aseptic loosening is suspected is not well defined. Intraoperative histology and cultures from periprosthetic tissue of 61 revision arthroplasties performed owing to suspected aseptic loosening were retrospectively reviewed. Frozen sections were evaluated following Mirra's criteria (adapted by Feldman). Culture was considered positive when the same microorganism was isolated in at least two samples. The cultures were positive in 12 cases and coagulasenegative staphylococci were the most common microorganisms (11 cases). In six out of 12 cases (50\%), the histology revealed more than five polymorphonuclear leukocytes per high-power field. The sensitivity, specificity, positive and negative predictive value of histology to detect the presence of microorganisms was 50, 81, 40 and $86 \%$, respectively. In conclusion, intraoperative histology using Mirra's criteria had a low sensitivity to predict the presence of microorganisms in samples from suspected aseptic prosthetic loosening. Modern Pathology (2006) 19, 874-877. doi:10.1038/modpathol.3800606; published online 7 April 2006
\end{abstract}

Keywords: aseptic loosening; prosthesis; infection; frozen sections

To establish the correct diagnosis of joint prosthesis loosening (septic or aseptic) is very important when choosing the most appropriate surgery. The typical symptoms such as redness, swelling and fever are usually absent. Different tests can be performed before surgery like C-reactive protein (CRP), erythrocyte sedimentation rate (ESR) and different radiological techniques (scintigraphy or computed tomography) as to obtain a final diagnosis, but these tests usually have low sensitivity and specificity. Therefore, in many cases, the definitive diagnosis is only achieved at the time of intervention. Only two intraoperative tests, Gram stain and histology, give

Correspondence: Dr G Bori, MD, Department of Orthopaedic and Traumatology, Hospital Clínic, Universidad de Barcelona, C/Villarroel, 170, 08036 Barcelona, Spain.

E-mail: gbori@clinic.ub.es or

Dr A Soriano, Department of Infectious Diseases, Hospital Clínic, University of Barcelona, Barcelona, Catalonia, Spain.

E-mail: asoriano@clinic.ub.es

Received 23 February 2006; revised 6 March 2006; accepted 10 March 2006; published online 7 April 2006 immediate information about the etiology of the prosthesis loosening. However, Gram stain sensitivity is lower than $30 \% .{ }^{1}$ The detection of more than five polymorphonuclear leukocytes (PMN) in intraoperative frozen sections of periprosthetic tissue has a high sensitivity and specificity. ${ }^{1-5}$ The number of revision arthroplasties has increased in the last years and the infection rate is higher than in primary arthroplasties. To determine if this high infection rate is associated with previous implant infection, the majority of surgeons submit samples to the microbiology laboratory in order to discard the presence of an undetected infection. Surprisingly, often when the loosening is considered aseptic, microorganisms are isolated from deep samples. These findings led some authors ${ }^{6}$ to include in the new classification of prosthetic joint infections, the 'positive intraoperative culture' as a different entity. In fact, this situation is a chronic infection that has not been identified by usual diagnostic tests such as histology of periprosthetic tissue. The usefulness of histology has not been evaluated in depth in the 
literature in those patients with prosthetic loosening without evident signs of infection (aseptic). For this reason, we retrospectively reviewed intraoperative histology and cultures in 102 patients who underwent a revision arthroplasty for preoperative diagnosis of aseptic loosening.

\section{Materials and methods}

From January 1998 until February 2005, the results of intraoperative histology of periprosthetic tissue and the cultures of intraoperative samples of 102 revision arthroplasties for suspected aseptic loosening were retrospectively studied.

The samples for the histological study were obtained from periprosthetic membranes or tissue suspected to be infected. The frozen sections of samples were studied using hematoxilin-eosin stain and based on Mirra's criteria (adapted by Feldman). ${ }^{2,3}$ The result was considered positive for infection if there were more than five PMN per high-power field in at least five separate microscopic fields $(\times 40) .{ }^{3}$ In addition, at least two periprosthetic tissue samples from different sites were submitted to the microbiology laboratory. These samples were always taken before the administration of antibiotic prophylaxis. The culture was considered positive when the same microorganism was isolated in two or more samples.

Prosthetic joint loosening was preoperative classified as aseptic or septic regarding clinical (pain, inflammatory signs, fistula) and/or biochemical (CRP, ERS) and/or radiological data (simple X-ray, Technetium $^{99 m}$ methylene diphosphonate scintigraphy and Tecnetium ${ }^{99 m}$ hexamethylpropyleneamineoxine-labelled leukocytes scintigraphy) and/ or microbiology results (culture of synovial fluid obtained by joint aspiration).

\section{Statistical Analysis}

The specificity, sensitivity, positive and negative predictive value of intraoperative histology was determined using the isolation of a microorganism in periprosthetic tissue as a gold standard.

\section{Results}

The characteristics of patients included are shown in Table 1. A total of 102 revision hip arthroplasties were performed during the study period. Forty-one were excluded because they only had one intraoperative culture. The intraoperative histology and culture results of the remaining 61 patients are shown in Table 2. In all cases, there were no macroscopic signs of infection and the result of intraoperative histology coincided with permanent sections of the same tissue. In 12 cases, a microorganism was identified at least in two samples
Table 1 Characteristics of patients with aseptic loosening of hip prosthesis

Aseptic loosening

\begin{tabular}{lc}
\hline Age (mean, range) & $74.3(52-90)$ \\
Male/female & $28 / 33$ \\
No. of cultures (mean, range) & $4(2-9)$ \\
Mean CRP (mg/dl) & 0.91 \\
Mean ESR (mm/h) & 21.5 \\
\hline
\end{tabular}

CRP, C-reactive protein (normal value: $<1 \mathrm{mg} / \mathrm{dl}$ ); ESR, erythrocyte sedimentation rate (normal value: $<20 \mathrm{~mm} / \mathrm{h}$ ).

Table 2 Frozen section and culture results in 61 patients with aseptic loosening

\begin{tabular}{lccc}
\hline Frozen section $^{\mathrm{a}}$ & \multicolumn{2}{c}{ Culture } & Total \\
\cline { 2 - 3 } & Positive & Negative & \\
\hline Positive & 6 & 9 & 15 \\
Negative & 6 & 40 & 46 \\
Total & 12 & 49 & \\
\hline
\end{tabular}

${ }^{\mathrm{a}}$ The result was considered positive for infection if there were more than five PMN per high-power field $(\times 40)$ in at least five separate microscopic fields.

(19.6\%), coagulase-negative staphylococci in 11 and Peptococcus sp in one. In only six out of 12 patients with positive culture, the pathologist identified more than five PMN per high-power field (forty times) in at least five separate microscopic fields in periprosthetic tissue. Ten out of 12 were treated with antibiotics for 3 months, and after 24 months of follow-up, they were asymptomatic. The sensitivity, specificity, positive and negative predictive value of histology to predict the presence of a microorganism in those patients with a suspected aseptic loosening was $50,81,40$ and $86 \%$, respectively.

The sensitivity, specificity, positive predictive value, negative predictive value and the gold standard used to define infection of the main articles that evaluate the usefulness of intraoperative histology are summarized in Table 3.

\section{Discussion}

The number of revision arthroplasties is increasing each year. In fact, in some countries, this procedure represents around $15 \%$ of annually implanted prosthesis. Therefore, it is necessary to improve diagnostic techniques in order to achieve a correct final diagnosis that may help surgeons not only to decide the best operative technique, but also to gain insight into the pathogenesis of joint prosthesis loosening. Up to now, there are no tests with high sensitivity and specificity in order to establish the correct preoperative diagnosis of prosthesis loosening, 
Table 3 Summary of the main articles

\begin{tabular}{|c|c|c|c|c|c|c|c|}
\hline Article & $\mathrm{N}$ & $\mathrm{S}(\%)$ & $\operatorname{Sp}(\%)$ & $P P V(\%)$ & $N P V(\%)$ & $P M N^{\mathrm{a}}$ & Gold standard \\
\hline Mirra et $a l^{2}$ & 34 & 100 & 98 & - & - & $>5$ & Cultures \\
\hline Feldman et $a l^{3}$ & 33 & 100 & 96 & - & - & $>5$ & Cultures \\
\hline Abdul-Karim et al ${ }^{8}$ & 64 & 43 & 97 & - & - & $>5$ & Histology and cultures \\
\hline Lonner et $a l^{5}$ & 175 & 84 & 96 & 70 & 98 & $>5$ & Cultures \\
\hline Spangehl et $a l^{1}$ & 202 & 80 & 94 & 74 & 96 & $>5$ & Histology and cultures \\
\hline Pons et $a l^{4}$ & 83 & 100 & 98 & 94 & 100 & $>5$ & Histology and cultures \\
\hline Banit et $a l^{11}$ & 121 & 67 & 93 & 67 & 93 & $>10$ & Cultures \\
\hline Musso et $a l^{12}$ & 45 & 50 & 95 & 60 & 92 & $>5$ & Cultures \\
\hline Ko et $a l^{10}$ & 40 & 67 & 97 & 86 & 91 & $>5$ & Cultures \\
\hline Bori et al (present article) & 61 & 50 & 81 & 40 & 86 & $>5$ & Cultures \\
\hline Athanasou et $a l^{17}$ & 106 & 90 & 96 & 88 & 98 & $>1$ & Cultures \\
\hline
\end{tabular}

$N$, number of cases analyzed; $S$, sensitivity; Sp, specificity; PPV, positive predictive value; NPV, negative predictive value; PMN, polymorphonuclear leukocytes.

${ }^{\mathrm{a}}$ The result was considered positive for infection if there were more than one, five or 10 PMN per high-power field $(\times 40)$ in at least five separate microscopic fields.

and in many cases the definitive diagnosis is not achieved until the examination of periprosthetic tissue. Histology has been considered the most useful test to establish a correct diagnosis; however, the systematic culture of periprosthetic tissue and a better management of this samples (inoculation into blood culture flasks ${ }^{7}$ ) have demonstrated that many preoperative aseptic loosening were indeed septic. In these patients, our results showed that intraoperative histology had a low sensitivity $(50 \%)$. It is of note that the highest sensitivity and specificity was achieved in those studies using as a gold standard not only the microbiological results, but also the result of permanent histology. As the correlation between intraoperative and permanent histology is over $95 \%,,^{3,8,9}$ it is evident that the diagnostic test (intraoperative histology) is included in the criteria to establish the final diagnosis of infection, therefore, hindering the correct evaluation of sensitivity, specificity, positive and negative predictive values. ${ }^{1,4}$ The present study and the more recently published articles ${ }^{8,10-12}$ that used as gold standard of infection the presence of microorganisms in periprosthetic tissue coincide about the lower values of sensitivity (Table 3 ). The problem is the pathogenic significance of intraoperative cultures in the absence of clinical symptoms and inflammatory signs in periprosthetic tissue. Recently, Tunney et $a l^{13}$ studied samples obtained after sonicating 120 prosthesis, removed for suspected aseptic loosening. They observed bacteria by immunofluorescence microscopy in $63 \%$ of them and by polymerase chain reaction amplified bacterial DNA in $72 \%$. The bacteria were present either as single cells or in aggregates of up to 300 bacterial cells, demonstrating that these microorganisms were not contaminants. These findings support the hypothesis that aseptic loosening is because of a low-grade inflammatory response against biofilms adhered to prosthesis surface, impeding the osteointegration. In addition, Dupont et $a l^{14}$ demonstrated a higher percentage of infection rates after revision arthroplasty in those patients with a positive intraoperative culture (11\%) than in those with negative intraoperative culture (3\%). In contrast, several reports have previously documented a high success rate with one-stage exchange for prosthetic joint infection. ${ }^{15}$ This discrepancy could be related with the use of local and systemic antibiotics. At present, in order to rule out the possibility of contamination, the same microorganism should be isolated in at least two different samples. This accepted criterion was applied in our study; however, it was not followed by other authors. ${ }^{8,11,12}$

Finally, the most common microorganism in our study as in others was coagulase-negative staphylococci. It is of note that Mirra et $a l^{2}$ described the criteria of positive result ( $>5$ PMN per field) in infected cases because of Pseudomonas sp $(n=7)$, Escherichia coli $(n=3)$, Proteus sp $(n=4)$, Streptococcus sp $(n=2)$ and Staphylococcus aureus $(n=1)$ and only in one case was due to coagulase-negative staphylococci. This raises the question about the usefulness of histology when the etiology is a low virulent microorganism. Pace et $a l^{16}$ and Athanassou et $a l^{17}$ found $<5 \mathrm{PMN}$ in $40 \%$ of cases when coagulase-negative staphylococci and Propionibacterium acnes were isolated. In fact, Athanassou et $a l^{17}$ and Pandey et al, ${ }^{18,19}$ using one PMN as cutoff for infection, observed a sensitivity of $90 \%$. It is possible that in patients with aseptic loosening, lowering the cutoff point (ie more than one PMN per field) may improve the sensitivity of intraoperative histology.

In conclusion, intraoperative histology, using Mirra's criteria, had a low sensitivity to predict the presence of microorganisms in samples from suspected aseptic prosthesis loosening.

\section{References}

1 Spangehl MJ, Masri BA, O’Connell JX, et al. Prospective analysis of preoperative and intraoperative 
investigations for the diagnosis of infection at the sites of two hundred and two revision total hip arthroplasties. J Bone Joint Surg Am 1999;81:672-683.

2 Mirra JM, Amstutz HC, Matos M, et al. The pathology of the joint tissues and its clinical relevance in prosthesis failure. Clin Orthop Relat Res 1976;117: 221-240.

3 Feldman DS, Lonner JH, Desai P, et al. The role of intraoperative frozen sections in revision total joint arthroplasty. J Bone Joint Surg Am 1995;77:1807-1813.

4 Pons M, Angles F, Sanchez C, et al. Infected total hip arthroplasty - the value of intraoperative histology. Int Orthop 1999;23:34-36.

5 Lonner JH, Desai P, Dicesare PE, et al. The reliability of analysis of intraoperative frozen sections for identifying active infection during revision hip or knee arthroplasty. J Bone Joint Surg Am 1996;78:1553-1558.

6 Tsukayama DT, Estrada R, Gustilo RB. Infection after total hip arthroplasty. A study of the treatment of one hundred and six infections. J Bone Joint Surg Am 1996;78:512-523.

7 Levine BR, Evans BG. Use of blood culture vial specimens in intraoperative detection of infection. Clin Orthop Relat Res 2001;382:222-231.

8 Abdul-Karim FW, McGinnis MG, Kraay M, et al. Frozen section biopsy assessment for the presence of polymorphonuclear leukocytes in patients undergoing revision of arthroplasties. Mod Pathol 1998;11: 427-431.

9 la Valle CJ, Bogner E, Desai P, et al. Analysis of frozen sections of intraoperative specimens obtained at the time of reoperation after hip or knee resection arthroplasty for the treatment of infection. J Bone Joint Surg Am 1999;81:684-689.

10 Ko PS, Ip D, Chow KP, et al. The role of intraoperative frozen section in decision making in revision hip and knee arthroplasties in a local community hospital. J Arthroplasty 2005;20:189-195.

11 Banit DM, Kaufer H, Hartford JM. Intraoperative frozen section analysis in revision total joint arthroplasty. Clin Orthop Relat Res 2002;401:230-238.

12 Musso AD, Mohanty K, Spencer-Jones R. Role of frozen section histology in diagnosis of infection during revision arthroplasty. Postgrad Med J 2003;79:590-593.

13 Tunney MM, Patrick S, Curran MD, et al. Detection of prosthetic hip infection at revision arthroplasty by immunofluorescence microscopy and PCR amplification of the bacterial $16 \mathrm{~S}$ rRNA gene. J Clin Microbiol 1999;37:3281-3290.

14 Dupont JA. Significance of operative cultures in total hip arthroplasty. Clin Orthop Relat Res 1986;211: 122-127.

15 Langlais F. Can we improve the results of revision arthroplasty for infected total hip replacement? J Bone Joint Surg Br 2003;85:637-640.

16 Pace TB, Jeray KJ, Latham Jr JT. Synovial tissue examination by frozen section as an indicator of infection in hip and knee arthroplasty in community hospitals. J Arthroplasty 1997;12:64-69.

17 Athanasou NA, Pandey R, de SR, et al. The role of intraoperative frozen sections in revision total joint arthroplasty. J Bone Joint Surg Am 1997;79:1433-1434.

18 Pandey R, Drakoulakis E, Athanasou NA. An assessment of the histological criteria used to diagnose infection in hip revision arthroplasty tissues. J Clin Pathol 1999;52:118-123.

19 Pandey R, Berendt AR, Athanasou NA. Histological and microbiological findings in non-infected and infected revision arthroplasty tissues. The OSIRIS Collaborative Study Group. Oxford Skeletal Infection Research and Intervention Service. Arch Orthop Trauma Surg 2000;120:570-574. 